\title{
DIE REAKTION VON AZOESTERN MIT TETRAALKYL-TETRAZENEN-(2)
}

\author{
F. EFFENBERGar und P. Fischer* \\ Institut für Organische Chemie der Universität Stuttgart
}

(Received in Germany 2 March 1970; Received in the UK for publication 13 March 1970)

Zusammenfasab-Bei der Umsetzung von Tetraalkyl-tetrazenen-(2) mit Azodicarbonsäure-diestern erfolgt primăr Angriff am Dialkylamino-stickstoff des Tetrazens. Das gebildete Betain 3 geht durch intramolekulare Protonenabstraktion in das Ylid 4 über, das sich zu 5 umlagert. Die Kinetik der Reaktion wird UV-spektroskopisch verfolgt und der Mechanismus gedeutet.

Abotract-Tetraalkyl-(2)-tetrazenes are attacked by azodicarboxylates at the dialkylamino nitrogen. By intramolecular proton abstraction, the betain 3 first formed is converted into the ylide 4 which then rearranges into 5. Kinetics of the reaction are investigated by UV spectroscopy; a mechanism is proposed.

KOMBINATION der drei möglichen mesomeren Grenzstrukturen nach der VB-Methode lässt für Tetrazene-(2) erhöhte Ladungsdichte an den Azo-Stickstoffatomen erwarten :

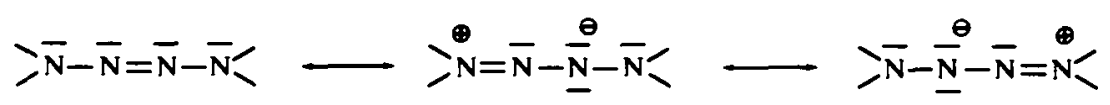

Tetrazene sollten daher bei DIELS-ALDER-Reaktionen mit inversem Elektronenbedarf als Dienophile reagieren. Entsprechende Umsetzungen sind jedoch gescheitert. ${ }^{1}$

Betrachtet man Tetrazene als planare Vier-Zentren- $\pi$-Systeme mit sechs $\pi$-Elektronen und nimmt für die freien Elektronenpaare an $\mathbf{N}_{2}$ und $\mathrm{N}_{3}$ völlige Orthogonalität an, so folgt aus einer einfachen HMO-Rechnung $\dagger$ (h-Werte: 06 für Azostickstoff $\mathrm{N}_{2,3}{ }^{2} 10$ für Dialkylamino-Stickstoff; ${ }^{3}$ alle k-Werte gleich 1.0), dass alle sechs $\pi$-Elektronen in bindenden Molekülorbitalen (-5.524, $-3.584,-0.603 \mathrm{eV})$ untergebracht sind.

Ladungsdichte im Grundzustand

Ladungsdichto im obersten besetzten Orbital

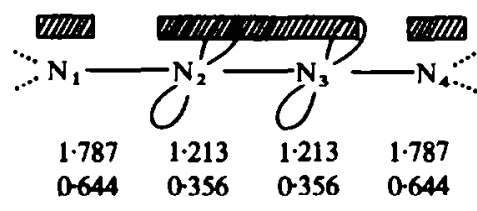

Die Ladungsdichteverteilung im Grundzustand und im obersten besetzten Orbital lässt einen elektrophilen Angriff nur an $\mathrm{N}_{1}$ und $\mathrm{N}_{4}$ erwarten. Dies wird durch die experimentellen Befunde bestătigt, die elektrophile Reaktion nur am DialkylaminoStickstoff zeigen, im allgemeinen unter anschliessender Sprengung der StickstoffViererkette. ${ }^{4.5}$

- Diplomarbeit P. Fischer, Univ. Stuttgart 1968

† Das Programm hat uns freundlicherweise Herr Dr. U. Sommer, Institut fur Physikalische Chemie der Universităt Stuttgart, zur Verfugung gestellt. 
Umsetzungen mit elektronenarmen Doppelbindungen sollten weitergehende Aussagen über das Reaktionsverhalten der Tetrazene-(2) erlauben, da hierbei neben einem elektrophilen Angriff an $\mathrm{N}_{1}$ bzw. $\mathrm{N}_{4}$ die Möglichkeit zu 2+2- und $2+4$ Cycloadditionen besteht. Wir haben deshalb die Reaktion von Azoestern mit Tetrazenen-(2) untersucht.

\section{ERGEBNISSE}

Beim Zusammengeben der Reaktionspartner 1 und 2 in Substanz tritt primär eine tief weinrote Farbe auf, die, je nach Substituenten $\mathbf{R}$ bzw. R', langsamer oder schneller verschwindet unter Bildung der schwach gelblichen, hochviskosen Verbindungen 5. Neben der von McBride et al. ${ }^{6}$ beschriebenen Umsetzung mit Tetranitromethan ist dies die erste Reaktion an Tetrazenen-(2), in deren Verlauf die Stickstoffkette erbalten bleibt.* Die Struktur der Produkte 5 ist durch ${ }^{1}$ H-NMR-Spektren (Tab. 1), Elementaranalysen und Molekulargewichte (Tab. 2) gesichert. Die UV-Spektren (Tab. 2) zeigen die charakteristische Doppelbandenstruktur der Tetrazene-(2) geringfügig (3-5 $\mathrm{m \mu}) \mathrm{zu}$ kürzeren Wellenlängen verschoben. Die IR-Spektren lassen sich bis auf eine sehr starke $\mathrm{N}-\mathrm{H}-$ Valenz-Schwingungsbande bei $3300 \mathrm{~cm}^{-1}$ und
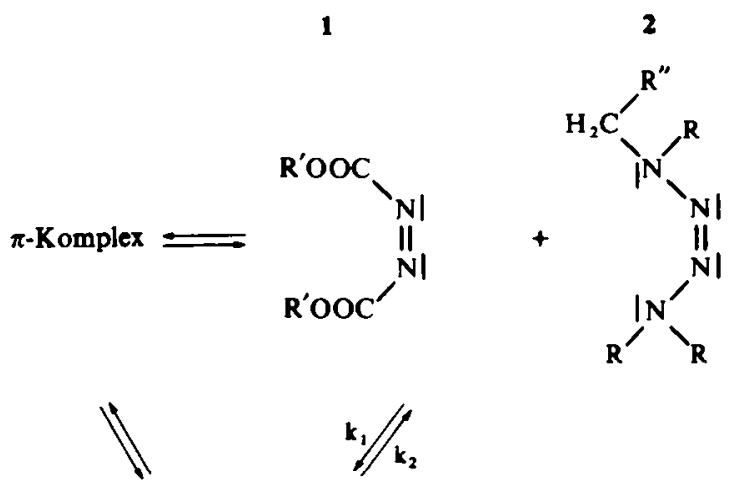

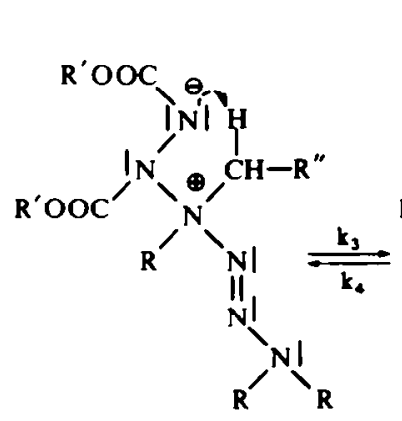

3

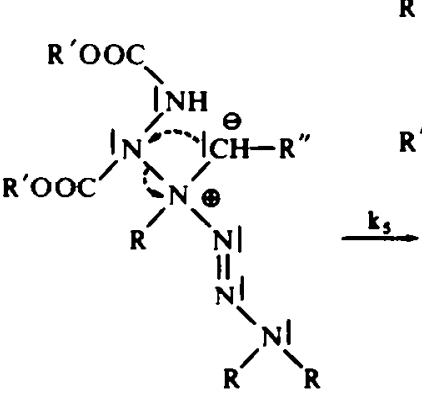

4<smiles>[R]OC(=O)NN(C([R])=O)C([R])N([R])/N=N/N([R])[R]</smiles>

$\mathbf{5}$
5a: $\mathbf{R}=\mathbf{R}^{\prime}=\mathrm{CH}_{3}, \mathbf{R}^{\prime \prime}=\mathbf{H}$
b: $R=\mathrm{CH}_{3}, \mathrm{R}^{\prime}=\mathrm{C}_{2} \mathrm{H}_{5}, \mathrm{R}^{\prime \prime}=\mathrm{H}$
c: $R=\mathrm{C}_{2} \mathrm{H}_{5}, \mathrm{R}^{\prime}=\mathrm{R}^{\prime \prime}=\mathrm{CH}_{3}$
d: $R=R^{\prime}=C_{2} H_{5}, R^{\prime \prime}=C_{3}$

- In jüngster Zeit ist auch die Oxidation der Alkylseitenketten zu Formyl-tetrazenen beschrieben.’ 
TABELLE 1. ${ }^{1}$ H-NMR-SPEETREN DER AdDUKTE 5 (1.0 mOLAR IN DCCl 3 , TMS ALS INT. STANDARd)

\begin{tabular}{|c|c|c|c|c|c|}
\hline Addukt & Gruppe & Signal & $\tau$ & Intensitāt & $J(\mathrm{~Hz})$ \\
\hline \multirow[t]{4}{*}{$5 n$} & $-\mathrm{N}-\underline{\underline{\mathrm{CH}}}_{3}$ & $\begin{array}{l}\text { Singulett* } \\
\text { Singulett* }\end{array}$ & $\begin{array}{l}7 \cdot 190^{d} \\
7 \cdot 165^{d}\end{array}$ & $9-0$ & \\
\hline & $-\mathrm{O}-\mathrm{CH}_{3}$ & Singulett & $\begin{array}{l}6.285^{\circ} \\
6.240^{\circ}\end{array}$ & 6.0 & \\
\hline & $-\mathrm{CH}_{2}-$ & Singulett & 4.93 & 1.8 & \\
\hline & $-\mathbf{N H}-$ & breites Signal um & $2 \cdot 7^{\circ}$ & $0-9$ & \\
\hline \multirow[t]{4}{*}{$\mathbf{5 b}$} & $-\mathrm{O}-\mathrm{CH}_{2}-\mathrm{CH}_{3}$ & $\begin{array}{l}\text { Triplett }^{\circ} \\
\text { Triplett }^{\circ}\end{array}$ & $\begin{array}{l}8 \cdot 740^{\prime} \\
8 \cdot 720^{S}\end{array}$ & 6.0 & 7.2 \\
\hline & $-\mathrm{N}-\mathrm{CH}_{3}$ & $\begin{array}{l}\text { Singulett } \\
\text { Singulett }\end{array}$ & $\begin{array}{l}7 \cdot 175^{d} \\
7 \cdot 145^{\prime}\end{array}$ & $9 \cdot 2$ & \\
\hline & $-\mathrm{O}-\mathrm{CH}_{2}-\mathrm{CH}_{3}$ & $\begin{array}{l}\text { Quadruplett } \\
\text { Quadruplett }^{b}\end{array}$ & $\begin{array}{l}5.815^{\prime} \\
5 \cdot 780^{\prime}\end{array}$ & $4 \cdot 0$ & $7 \cdot 15$ \\
\hline & $-\mathrm{CH}_{2}-$ & $\begin{array}{l}\text { Singulett } \\
\text { breites Signal }\end{array}$ & $\begin{array}{l}4.915 \\
3.07^{f}\end{array}$ & $\begin{array}{l}1.9 \\
0.7\end{array}$ & \\
\hline \multirow[t]{6}{*}{ Sc } & $-\overline{\mathrm{N}}-\mathrm{CH}_{2}-\mathrm{CH}_{3}$ & Triplett & 8.890 & $9 \cdot 0$ & $7 \cdot 0$ \\
\hline & $>\mathrm{CH}-\mathrm{CH}_{3}$ & $\begin{array}{l}\text { Dublett } \\
\text { Dublett }\end{array}$ & $\begin{array}{l}8.465 \\
8 \cdot 405\end{array}$ & $\begin{array}{l}2.0 \\
1.0\end{array}$ & 6.5 \\
\hline & $-\mathrm{N}-\mathrm{CH}_{2}-\mathrm{CH}_{3}$ & Quadruplett & $6.730^{\circ}$ & $6 \cdot 2$ & $7 \cdot 0$ \\
\hline & $-\mathrm{O}-\mathrm{CH}_{3}$ & $\begin{array}{l}\text { Singulett } \\
\text { Singulett }^{b}\end{array}$ & $\begin{array}{l}6.265^{\circ} \\
6.250^{\circ}\end{array}$ & $5 \cdot 9$ & \\
\hline & $2 \mathrm{CH}-\mathrm{CH}_{3}$ & Quadruplett & $4 \cdot 305$ & $0-6$ & 6.4 \\
\hline & $-\mathrm{NH}-$ & breites Signal um & $3 \cdot 4^{e}$ & 0.5 & \\
\hline \multirow[t]{7}{*}{ sa } & $-\mathrm{N}-\mathrm{CH}_{2}-\mathrm{CH}_{3}$ & Triplett & $8.885^{\circ}$ & $9 \cdot 0$ & $7 \cdot 15$ \\
\hline & $-\mathrm{O}-\mathrm{CH}_{2}-\mathrm{CH}_{3}$ & $\begin{array}{l}\text { Triplett } \\
\text { Triplett }\end{array}$ & $\begin{array}{l}8 \cdot 760^{\circ} \\
8.745^{\circ}\end{array}$ & 60 & $7 \cdot 10$ \\
\hline & $\geq \mathrm{CH}-\mathrm{CH}_{3}$ & $\begin{array}{l}\text { Dublett } \\
\text { Dublett }^{c}\end{array}$ & $\begin{array}{l}8 \cdot 460 \\
8 \cdot 410\end{array}$ & $3 \cdot 0$ & 6.65 \\
\hline & $-\mathrm{N}-\mathrm{CH}_{2}-\mathrm{CH}_{3}$ & Quadruplett & $6.735^{\circ}$ & 6.0 & $7 \cdot 10$ \\
\hline & $-\mathrm{O}-\overline{\mathrm{CH}}_{2}-\mathrm{CH}_{3}$ & $\begin{array}{l}\text { Quadruplett } \\
\text { Quadruplett }\end{array}$ & $\begin{array}{l}5.825^{J} \\
5 \cdot 800^{S}\end{array}$ & 3.8 & $7 \cdot 10$ \\
\hline & $=\mathrm{CH}-\mathrm{CH}_{3}$ & Quadruplett & $4 \cdot 285$ & 0.8 & 6.65 \\
\hline & $-\mathbf{N H}-$ & breites Signal um & $3 \cdot 5^{\prime}$ & 0.8 & \\
\hline
\end{tabular}

“ Ob die geringfügige Aufspaltung des $\mathrm{N}-\mathrm{CH}_{3}$-Signals etwa im Verhăltnis 1:2 auf unterschiedlicher chemischer Verschiebung oder auf sterischer Hinderung beruht, ist nicht völlig sicher

- X̂hnliche geringe Aufspaltung der Esterprotonen-Signale finden wir auch bei phenyl-substituierten Hydrazoestern

- Die Aufspaltung des $\mathrm{CH}_{3}-\mathrm{CH}_{\mathrm{N}^{2}}^{-\mathrm{N}^{2}}$-Dubletts in zwei Signale kann auf eingefrorene Rotation oder auf das benachbarte Asymmetriezentrum zurückgeführt werden

d Tetramethyl-tetrazen-(2) $+7 \cdot 185$

- Hydrazodicarbonsăure-dimethylester $\tau 6 \cdot 22,3 \cdot 1$

J Hydrazodicarbonslure-diathylester $+8.72,5.765(\mathrm{~J} 7.1 \mathrm{~Hz}), 3.4$

- Tetraäthyl-tetrazen-(2) ₹ 8.895, 6.732 (J 7.1 Hz) 
eine Ester-Carbonyl-Bande bei $1710 \mathrm{~cm}^{-1}$ (Azoester $\sim 1780 \mathrm{~cm}^{-1}$ ) durch Superposition der Absorptionen von 1 und 2 deuten.

Die Identităt der Produkte bei Reaktion in Substanz und in Lösung ist NMRspektroskopisch gesichert. Beim Versuch, sie aus der Reaktionslösung durch Abziehen des Solvens zu isolieren, zersetzen sich die labilen Adduk.e 5 unter heftiger Gasentwicklung bereits bei $20^{\circ}$.

Tabellz 2. Molekulargewichtb UND UV-Absorptiongn der ADduktB 5

\begin{tabular}{|c|c|c|c|c|c|}
\hline \multirow[b]{2}{*}{ Sa } & \multirow{2}{*}{$\begin{array}{c}\begin{array}{c}\text { Molgewicht } \\
\text { berechnet }\end{array} \\
262 \cdot 3\end{array}$} & \multirow{2}{*}{\begin{tabular}{|c|} 
gefunden \\
$255\left(\mathrm{CHCl}_{3}\right)$ \\
$274\left(\mathrm{C}_{6} \mathrm{H}_{6}\right)$
\end{tabular}} & \multirow{2}{*}{$\frac{\lambda^{1}[\mathrm{m \mu}]\left(\varepsilon^{1} \mathrm{~mol}^{b}\right.}{271(8437)^{c}}$} & \multicolumn{2}{|c|}{$\lambda^{2}[\mathrm{~m} \mu]\left(\varepsilon^{2}{ }_{\mu_{0}}\right)^{\phi}$} \\
\hline & & & & 240 & $(3014)^{c}$ \\
\hline $5 b$ & $290-3$ & $290\left(\mathrm{CHCl}_{3}\right)$ & $272.5(6120)$ & 242 & $(2250)$ \\
\hline Sc & 318.4 & $\begin{array}{l}311\left(\mathrm{C}_{6} \mathrm{H}_{6}\right) \\
324\left(\mathrm{C}_{6} \mathrm{H}_{6}\right)\end{array}$ & $278.5(8454)^{4}$ & 242 & $(4057)^{2}$ \\
\hline 5d & $346 \cdot 4$ & $344\left(\mathrm{C}_{6} \mathrm{H}_{6}\right)$ & $278.5(8692)$ & 243.5 & $(4130)$ \\
\hline
\end{tabular}

asmometrisch bestimmt; ${ }^{\circ}$ in $\mathrm{CH}_{3} \mathrm{OH}$; ${ }^{c}$ Tetramethyl-tetrazen-(2) $276 \mathrm{m \mu}$ (7433), $243 \mathrm{m \mu}$ (2930);

'Tetraăthyl-tetrazen-12) $283 \mathrm{m \mu}$ (6664), $247 \mathrm{~m} \mu$ (4636)

In Anlehnung an den von Huisgen und Jakob für die Reaktion von N,N-Dimethylanilin mit Azoester vorgeschlagenen Mechanismus ${ }^{8}$ nehmen wir für die Umsetzung von 1 mit 2 die Reaktionsfolge $3 \rightarrow 4 \rightarrow 5$ an.

Die primäre Rotfärbung beruht wahrscheinlich auf der Bildung eines $\pi$-Komplexes. Ob 3 über den $\pi$-Komplex oder aus 1 und 2 direkt entsteht, kann nicht entschieden werden.

Zur Prüfung des vorgeschlagenen Reaktionsverlaufs haben wir die Kinetik der Reaktion untersucht.

\section{Kinetik der Reaktion}

Für die kinetische Untersuchung wird der besonders gut reagierende 9 Azodicarbonsäure-dimethylester eingesetzt. Dem vorgeschlagenen Verlauf der Umsetzung entspricht die mechanistische Folge

$$
\begin{aligned}
& 1+2 \underset{k_{2}}{\stackrel{k_{1}}{p_{2}}} \\
& 3{\stackrel{k_{3}}{k_{4}}}_{4} \\
& 4 \stackrel{k_{3}}{\rightarrow} 5
\end{aligned}
$$

Für den grössten Teil der Reaktion kann Quasi-Stationarität der Betain- und der Ylid-Konzentration angenommen werden $(\mathrm{d}[3] / \mathrm{d} t=0 ; \mathrm{d}[4] / \mathrm{d} t=0)$. Damit ergibt sich für die Änderung der Azoester-Konzentration:

$$
\frac{\mathrm{d}[1]}{\mathrm{d} t}=-\frac{k_{1} k_{3} k_{5}}{k_{2} k_{4}+\left(k_{2}+k_{3}\right) k_{5}}[1][2]
$$


Mit den beiden Quasistationaritătsbedingungen vereinfacht sich das Zeitgesetz zu :

$$
\frac{\mathrm{d}[1]}{\mathrm{d} t}=k_{1}[1][2]
$$

In Tabelle 3 sind die experimentell ermittelten Geschwindigkeitskonstanten für eine Reaktion 2 Ordnung zwischen Tetramethyl-tetrazen-(2)(TMT) bzw. Tetraäthyltetrazen-(2) (TÄT) mit Azodicarbonsäure-dimethylester zusammengestellt. Für die Aktivierungsenergie der Reaktion 2. Ordnung wird $9 \mathrm{kcal} / \mathrm{Mol}$ gefunden.

TABELLB 3. $k_{\mathrm{II}} \cdot 10^{3}$ [LTTER $\mathrm{MOL}^{-1} \mathrm{SBC}^{-1}$ ] BB $19.9 \pm 0.1^{\circ} \mathrm{C}$

\begin{tabular}{ccc}
\hline Lösungsmittel & TMT & TÄT \\
\hline $\mathrm{CCl}_{4}$ & 2.017 & 23.68 \\
$\mathrm{CH}_{3} \mathrm{CN}$ & 12.17 & 95.00 \\
$\mathrm{CHCl}_{3}$ & 35.67 & $351 \cdot 3^{\circ}$ \\
$\mathrm{CH}_{3} \mathrm{OH}$ & $450-8$ & $4829^{\circ}$ \\
\hline aie $\mathrm{k}_{\mathrm{n}}$-Werte geben in diesen Fullen nur bis etwa $1 / 3$ Umsatz \\
den experimentell gefundenen Verlauf der Reaktion wieder
\end{tabular}

In Methanol als Lösungsmittel wird bei TÄT für einen weiten Bereich der Reaktion (1/3 bis 4/5 Umsatz), in Chloroform für einen schmalen Bereich Reaktion 1. Ordnung gefunden. Die Reaktionsgeschwindigkeit hängt hierbei alleine von der Azoesterkonzentration ab, die Geschwindigkeitskonstante zeigt nur geringe Solvensabhăngigkeit :

$$
\begin{array}{ll}
k_{1} & 31.0 \times 10^{-5} \mathrm{Sec}^{-1} \text { in } \mathrm{CH}_{3} \mathrm{OH}\left(19.9 \pm 0.1^{\circ} \mathrm{C}\right) \\
k_{1} & 25.1 \times 10^{-5} \mathrm{Sec}^{-1} \text { in } \mathrm{HCCl}_{3}\left(19.9 \pm 0.1^{\circ} \mathrm{C}\right)
\end{array}
$$

\section{Diskussion der Ergebnisse}

McBride und Thun haben die Geschwindigkeitskonstanten der sauren Zersetzung verschiedener Tetraalkyl-tetrazene-(2) mit ihren $\mathrm{p} K_{\mathrm{b}}$-Werten korreliert. ${ }^{10}$ Die beste Gerade durch die Messpunkte ergibt für das Verhältnis der Geschwindigkeitskonstanten der Zersetzung von TMT und TÄT 1:10-11. (Experimentell wird von den Autoren 1:7.9 gefunden.) Da bei der Zersetzung mit Săure nur das Triazensystem b

$$
>\overline{\mathrm{N}}-\mathrm{N}=\mathrm{N}-\overline{\mathrm{N}}\left\langle+\mathrm{H}^{\bullet} \Rightarrow>_{\mathrm{N}}^{\bullet}-\mathrm{N}=\mathrm{N}-\overline{\mathrm{N}}<\right.
$$

zerfallen kann, ${ }^{4,10}$ ist die Lage des Protonierungsgleichgewichtex, die eine Funktion des $\mathrm{p} K_{\mathrm{b}}$-Wertes ist, entscheidend für die Zersetzungsgeschwindigkeit.

Die Zersetzungsgeschwindigkeit der Tetrazene-(2) mit HX-Verbindungen lässt sich durch einen Mechanismus wiedergeben, der dem der Reaktion von 1 mit 2 vollkommen entspricht. Der langsamste und damit geschwindigkeitsbestimmende Schritt ist die Protonierung bzw. die Bildung von 3. Alle folgenden Reaktionsschritte verlaufen rascher.

Die aufgezeigte Analogie erklärt auch unseren Befund, dass TMT in allen Lösungsmitteln etwa 10-11 mal rascher mit Azodicarbonsăure-dimethylester reagiert als TÄT. 
Die kurzwellige Tetrazen-UV-Bande ist einem Dreizentren- $\pi$-System zuzuordnen :1 1* ein Dialkylaminostickstoff muss durch Solvatation von direkter mesomerer Wechselwirkung mit dem restlichen $\pi$-System ausgeschlossen sein. Das Extinktionsverhăltnis der beiden Tetrazenbanden kann somit als Mass dienen für Basizităt bzw.

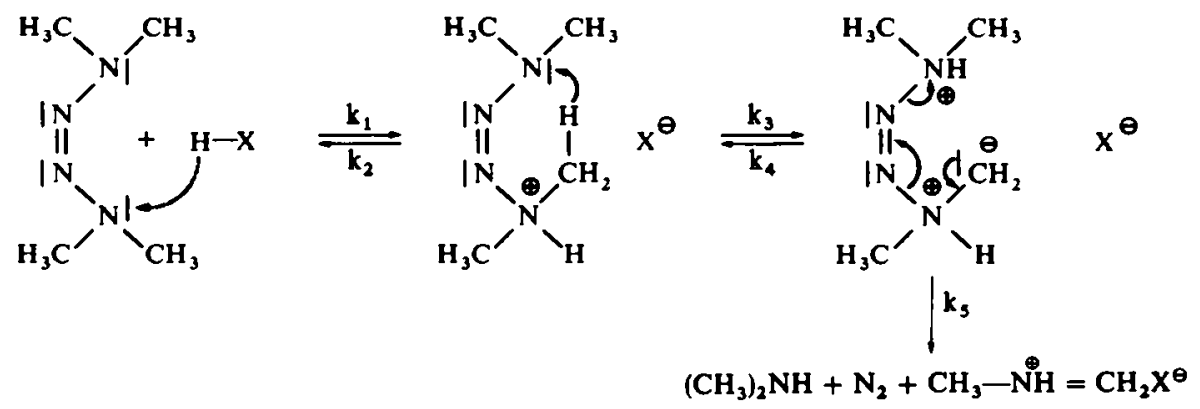

Nucleophilie des $\mathrm{NR}_{2}$-Stickstoffs (Tab. 4). Beim TMT zeigt $\varepsilon^{1} / \varepsilon^{2}$ im Gegensatz zu TÄT nur geringe Solvens-Abhängigkeit. Die experimentell gefundene gleiche Abstufung der Reaktionsgeschwindigkeit von TMT und TÄT in den einzelnen Lösungsmitteln lässt sich dann nur durch einen entscheidenden Solvens-Einfluss auf den Ubergangszustand im geschwindigkeitsbestimmenden Schritt deuten. Die Bildung des Betains 3 erfordert Ladungstrennung für die die Aktivierungsenergie in hochpolaren, mehr noch in Protonen-Donor-Lösungsmitteln herabgesetzt ist im Vergleich zu unpolaren Solventien. Daher wird für das stärker basische TÄT in $\mathrm{CHCl}_{3}$ und $\mathrm{CH}_{3} \mathrm{OH} \mathrm{k}_{1}$ so gross gegenüber $\mathrm{k}_{3}$, dass dem Betain 3 noch vor der Umlagerung in das Ylid 4 ein Proton durch freies TÄT entrissen wird (Geschwindigkeitskonstante $\mathbf{k}_{3}^{\prime}$ ). Aus einem entsprechend variierten Reaktionsschema folgt, wenn wiederum Quasi-Stationarităt für das Betain 3 angenommen wird, ohne weitere Annahmen direkt das Zeitgesetz

$$
-\frac{\mathrm{d}[1]}{\mathrm{d} t}=k_{3}^{\prime}[3][2]=k^{\prime}[2]
$$

also Reaktion 1. Ordung, wie sie auch experimentell gefunden wird. Für die normale Reaktion 2 Ordung kann somit bimolekulare Deprotonierung von 3 durch Tetrazen ausgeschlossen werden. Da der $\mathrm{p} K_{\mathrm{b}}$-Wert von Tetraisopropyl-tetrazen-(2) (TPT) sicher $\ll 60$ ist, ${ }^{10}$ sollte $k_{1} \gg k_{3}$ sein und somit die Zersetzung des Triazens der Umlagerung zu 4 den Rang ablaufen. Tatsächlich beobachtet man in Lösung wie in Substanz bei der Umsetzung von TPT mit Azoestern heftige Reaktion unter $\mathbf{N}_{2}$ Entwicklung, d.h. Zersetzung. Beim 1,4-Dimethyl-1,4-diphenyl-tetrazen-(2) dagegen ist die Basizităt so gering, dass die Reaktion nicht über eine $\pi$-Komplexbildung hinauskommt.

\section{$1: 2$-Addukte von Tetraalkyl-tetrazenen-(2) mit Azoestern}

Eine Lösung des Adduktes $5 \mathrm{a}$ in $\mathrm{CCl}_{4}$ reagiert mit Azodicarbonsãure-dimethylester innerhalb einer Woche, mit Azodicarbonsäure-diäthylester in sechs Wochen zu Bis-addukten. Ebenso wie bei den 1:1-Umsetzungsprodukten aus Tetrazenen und

- Dies folgt auch aus HMO-Berechnungen. 
Die Reaktion von Azoestern mit Tetraslkyl-tetrazenen-(2)

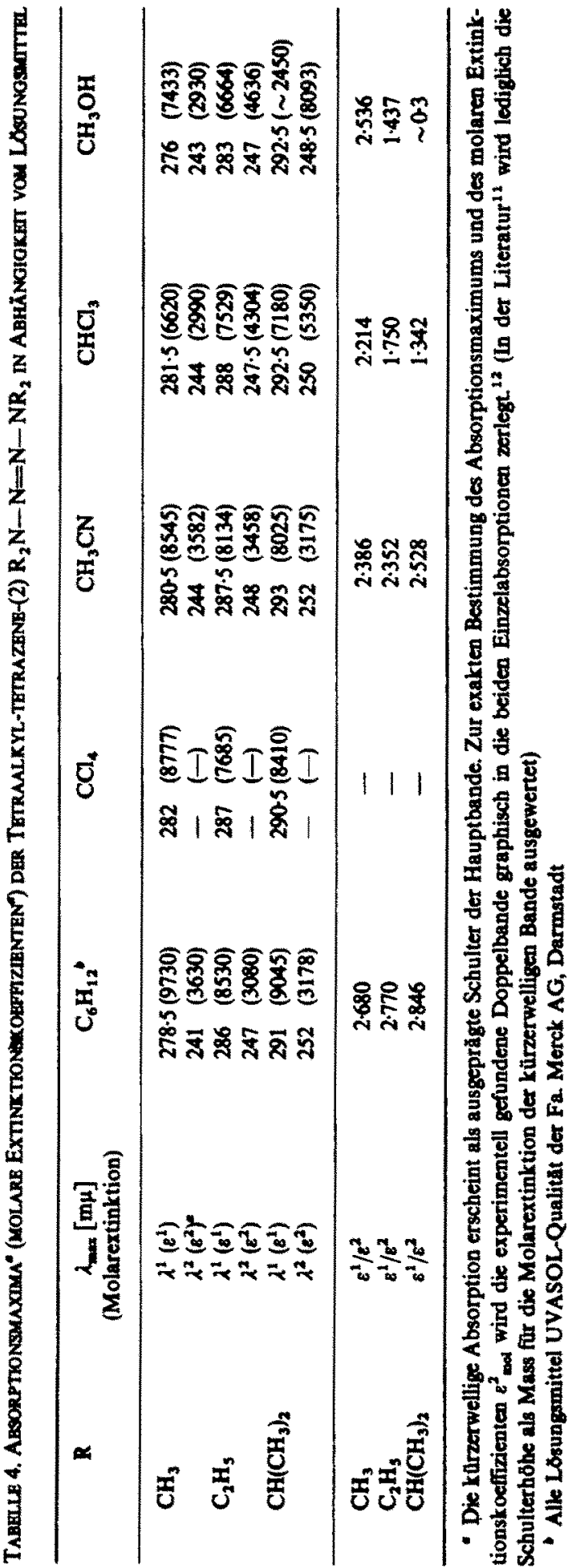


Azoester gelingt es auch hier nicht, die NMR-spektroskopisch identifizierten 1:2Addukte aus den Lösungen zu isolieren. In reiner Form kann ein 1:2-Addukt aus TÄT und Azodicarbonsăure-dimethylester bei Reaktion in Substanz erhalten werden, dem wir aufgrund des NMR-Spektrums die Struktur 6 zuordnen.<smiles>CCN(N=NN(CC)C(C)N(CC)C(C)NNC(=O)OC(C)=O)C(C)=O</smiles>

Die Signallagen sind identisch mit denen des 1:1-Adduktes 5c; das UV-Spektrum zeigt die gewohnte Tetrazenstruktur $\left(\lambda^{1} 277.5 \mathrm{m \mu} \lambda^{2} 243 \mathrm{m \mu}\right)$.

Mit anderen elektrophilen Doppelbindungssystemen wie TCÄ oder Diphenylketen erfolgt ebenfalls primär Reaktion an einem Dialkylamino-Stickstoff; die anschliessenden Folgereaktionen werden derzeit untersucht.

\section{EXPERIMENTALLER TEIL}

1. Tetra-isopropyl-tetrazen-(2). $28-4 \mathrm{~g}$ (0-245 Mol) 1,1-Diisopropylhydrazin ${ }^{13}$ werden in $75 \mathrm{ml}$ absol. Äther gelöst. Bei $0^{\circ}$ werden unter Rühren portionsweise $53.3 \mathrm{~g}$ gelbes $\mathrm{HgO}_{\mathrm{g}} \mathrm{zugegeben}$. Nach insgesamt 4 Stunden Reaktionszeit wird vom ausgeschiedenen $\mathrm{Hg}_{\mathrm{g}}$ abdekantiert, der Rückstand mehrmals mit absol. Äther gewaschen, die vereinigten Ẍtherextrakte getrocknet und fraktioniert destilliert Ausbeute: $19.5 \mathrm{~g}$ (70\% d.Th.), Sdp. 11 97-98 ${ }^{\circ}$, Schmp. $5^{\circ}$.

2. (1,3,3-Trimethyl-tetrazen-(2)-0-) (1,2-dicarbmethoxy-hydrazino-)methan (50). $1 \cdot 1617 \mathrm{~g}$ (0-01 Mol) Tetramethyl-tetrazen-(2) ${ }^{11}$ und $1.4610 \mathrm{~g}(0-01 \mathrm{Mol})$ Azodicarbonsăure-dimethylester werden ohne Losungsmittel zusammengegeben. Das Reaktionsgemisch wird tiefdunkelrot und erwarmt sich nach $30 \mathrm{Min}$ zunehmend. Nach 12 Stdn. ist Entfarbung unter Zunahme der Viskosităt eingetreten. Ausbeute: quant. $\left[\mathrm{C}_{8} \mathrm{H}_{18} \mathrm{~N}_{6} \mathrm{O}_{4}\right.$ (262.3) Ber: C, 36.63; H, 6.92; N, 32.05. Gef: C, 36.72; H, 6.84; N, 32.12\%].

3. (1,3,3-Trimethyl-tetrazen-(2)-o-) (1,2-dicarbahoxy-hydrazino-)methan (56). Die durch Zusammengeben von $1.161 \mathrm{~g}(0-01 \mathrm{Mol})$ Tetramethyl-tetrazen-(2) und $1.7416 \mathrm{~g}$ (0-01 Mol) Azodicarbonsare-diäthylester entstehende dunkelrote Reaktionsmischung zeigt nach 24 Stdn. merkliche Entfarbung und Viskositătszunahme. Nach 4 Tagen liegt ein fast farblosés, hochviskoses $\mathrm{Ol}$ vor. Ausbeute: quant. $\left[\mathrm{C}_{10} \mathrm{H}_{22} \mathrm{~N}_{6} \mathrm{O}_{4}(290-3)\right.$ Ber: C, $41.37 ; \mathrm{H}, 7.64 ; \mathrm{N}, 28.95$. Gef: C, $41.20 ; \mathrm{H}, 7.53 ; \mathrm{N}, 29.16 \%]$.

4. 1-(1,3,3-Triäthyl-tetrazen-(2)-o-)1-(1,2-dicarbmethoxy-hydrazino-) äthan (Sc). Die beim Zusammengeben von $0.8614 \mathrm{~g}(0-005 \mathrm{Mol})$ Tetraatthyl-tetrazen $-(2)^{11}$ und $0.7305 \mathrm{~g}(0-005 \mathrm{Mol})$ Azodicarbonsăure-dimethylester entstehende dunkelrote homogene Mischung erwårmt sich nach ca. 2 Min. sehr stark $\left(\sim 60^{\circ}\right)$; es wird deshalb mit Wasser gekublt. Nach 20 Min ist das Reaktionsgemisch orangefarben, nach 2 Stdn hellgelb, nach $24 \mathrm{Stdn}$. fast farblos und zähviskos. Ausbeute: quant. [ $\mathrm{C}_{12} \mathrm{H}_{26} \mathrm{~N}_{6} \mathrm{O}_{4}(318 \cdot 4)$ Ber: $\mathrm{C}, 45.27 ; \mathrm{H}, 8.23$; $\mathrm{N}, 26 \cdot 40$. Gef: C, $45 \cdot 02 ; \mathrm{H}, 8.21 ; \mathrm{N}, 26 \cdot 24 \%]$.

5. 1-(1,3,3-Triäthyl-tetrazen-(2)-o-)1-(1,2-dicarbäthoxy-hydrazino-)athan (5d). Beim Zusammengeben von $0.8614 \mathrm{~g}(0-005 \mathrm{Mol})$ Tetraăthyl-tetrazen-(2) und $0.8708 \mathrm{~g}(0-005 \mathrm{Mol})$ Azodicarbonsăure-diåthylester tritt nur måssige Rotfarbung auf. Nach $30 \mathrm{Min}$. wird Viskositătszunahme beobachtet und nach $24 \mathrm{Stdn}$. ist ein hellgelbes Oll entstanden. Ausbeute: quant. $\left[\mathrm{C}_{14} \mathrm{H}_{30} \mathrm{~N}_{6} \mathrm{O}_{4}(346.4) \mathrm{Ber}: \mathrm{C}, 48.54 ; \mathrm{H}, 8.73 ; \mathrm{N}, 24.26\right.$. Gef: C, $48 \cdot 36 ; \mathrm{H}, 8 \cdot 81 ; \mathrm{N}, 23 \cdot 93 \%$ ].

6. 1,4-Didthyl-1,4-bis-1-(1,2-dicarbmethoxy-hydrazino-)éthyl-tetrazen-(2) (6). $\mathrm{Zu} 1.4625 \mathrm{~g}$ (0-01 Mol) Azodicarbonshure-dimethylester werden unter guter Kuhlung $0-8646 \mathrm{~g}(0-005 \mathrm{Mol})$ Tetråthyl-tetrazen-(2) gegeben. Die anfanglich dunkelrote Reaktionslösung ist nach 24 Stdn. zu einer farblosen Masse erstarrt, die in abs. Ather gelöst wird. Aus der atherischen Losung fallen rasch $820 \mathrm{mg}$ feine weisse Kristalle aus, die mit Aceton aufgełocht und mit abs. Äther gewaschen werden. Reinausbeute: $670 \mathrm{mg} 29 \%$ farbl. Krist., Fp. 142.3 ${ }^{\circ}$ [ $\mathrm{C}_{16} \mathrm{H}_{32} \mathrm{~N}_{8} \mathrm{O}_{8}$ (464.5) Ber: C, 41.37; H, 6.94; N, 24.13. Gef: C, 41.58; H, 6.87; N, 23.89\%]. (Molekulargewicht ist wegen der geringen Loslichkeit osmometrisch nicht zu bestimmen.)

7. UV-Kinetik In einem Messkolbchen wird Tetrazen vorgelegt und zur Halfe mit Losungsmittel aufgefullt. Zur Zeit $t=0$ wird die squivalente Menge Azodicarbonslure-dimethylester zugegeben und 
rasch auf das Messvolumen aufgefullt. Die Reaktionslosung wird im Thermostaten $\left( \pm 0-1^{\circ}\right)$ gehalten. Jeweils zur Zeit $t$ wird eine Probe entnommen und zu soviel $L$ ssungsmittel gegeben, dass die Gesamtkon-

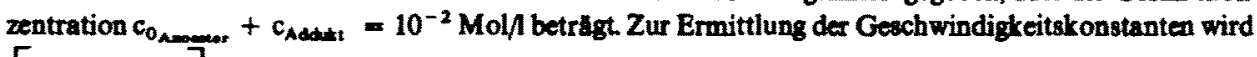
$\left[\frac{A^{\circ} \text { Amonerer }}{A_{\text {Amoner }}}-1\right]$ gegen $t$ aufgetragen.

\begin{tabular}{lcccc}
\multicolumn{5}{c}{ Konzentration der Reaktionsiossungen $[\mathrm{Mol} / 1]$} \\
$\mathrm{TMT}$ & $\mathrm{CCl}_{4}$ & $\mathrm{CH}_{3} \mathrm{CN}$ & $\mathrm{CHCl}_{3}$ & $\mathrm{CH}_{3} \mathrm{OH}$ \\
$\mathrm{TAT}$ & $10^{\circ}$ & $10^{\circ}$ & $10^{\circ}$ & $10^{-1}$ \\
& $10^{\circ}$ & $10^{\circ}$ & $10^{-1}$ & $10^{-2}$
\end{tabular}

Danksagung-Der Deutschen Forschungagemeinschaft und dem Fonds der Chemischen Industrie gilt unser Dank für Sachbeihilfen. P. Fischer dankt der Studienstiftung des Deutschen Volkes für ein Stipendium.

\section{LITERATUR}

${ }^{1}$ D. Mackay und W. A. Waters, J. Chem. Soc. C, 813 (1966)

${ }^{2}$ F. Yoneda, K. Suzuki und Y. Nitta, J. Org. Chem. 32, 727 (1967)

${ }^{3}$ A. Zweig, J. E. Lancaster, M. T. Neglia und W. H. Jura, J. Am. Chem. Soc. 86,4130 (1964)

4. Wieland und H. Fressel, Liebigs Ann 392, 127 (1912)

5 C. J. Michejda und D. Romans, Tetrahedron Letters 4213 (1969)

- W. E. Thun, D. W. Moore und W. R. McBride, J. Org. Chem. 31, 923 (1966)

7 W. E. Thun und W. R. McBride, Ibid 34, 2997 (i969)

- R. Huisgen und F. Jakob, Liebigs Ann 590,37 (1954)

- E. Fahr und H. Lind, Angew. Chem. 72376 (1966)

${ }^{10}$ W. R. McBride und W. E. Thun, Inorg. Chem. 5, 1846 (1966)

11 W. E. Bulh, J. A. Seaton und L. F. Audrieth, J. Am Chem. Soc. 90,2516 (1958)

12 H. H. Jaffe und M. Orchin, Theory and Application of Ultraviolet Spectroscopy S. 111. Wiley, New York (1965)

${ }^{13}$ D. M. Lemal, F. Menger und E. Coats, J. Am. Chem. Soc. 86, 2395 (1964) 